\title{
A study on dynamics of mechanical systems including joints with clearance and lubrication
}

\author{
P. Flores ${ }^{\mathrm{a}, *}$, J. Ambrósio ${ }^{\mathrm{b}}$, J.C.P. Claro ${ }^{\mathrm{a}}$, H.M. Lankarani ${ }^{\mathrm{c}}$, C.S. Koshy ${ }^{\mathrm{c}}$ \\ a Departamento de Engenharia Mecânica, Universidade do Minho Campus de Azurém, 4800-058 Guimarães, Portugal \\ ${ }^{\mathrm{b}}$ Instituto de Engenharia Mecânica (IDMEC), Instituto Superior Técnico, Av. Rovisco Pais, 1049-001 Lisboa, Portugal \\ ${ }^{\mathrm{c}}$ Department of Mechanical Engineering, Wichita State University, Wichita, KS 67260-133, USA
}

Received 13 September 2004; received in revised form 20 September 2005; accepted 10 October 2005

Available online 18 November 2005

\begin{abstract}
In this work a methodology for the dynamical analysis of mechanical systems considering realistic joint characteristics, namely, joints with clearance and lubrication is presented. For the case of the joints with clearance modelled as a contact pair with dry contact, a technique using a continuous approach for the evaluation of the contact force is applied, in which the energy dissipation in form of hysteresis damping is considered. The friction forces are calculated using a modified Coulomb's friction law. For the lubricated case, the hydrodynamic theory for dynamically loaded journal-bearings is used to compute the forces generated by lubrication action. The numerical results point out that the existence of dry joint clearances causes high peaks on the kinematic and dynamic system's characteristics due to contact-impact forces when compared to those obtained with lubricated model. The performance of the lubricated joint is closer to that of an ideal joint. (C) 2005 Elsevier Ltd. All rights reserved.
\end{abstract}

Keywords: Joint clearance; Lubrication; Contact forces; Hydrodynamic forces; Multibody dynamics

\section{Introduction}

A mechanical system is made of several components, which can be divided in two major groups, namely, links, that is, bodies with a convenient geometry, and joints, which introduce some restrictions on the relative motion of the various bodies of the system. Usually, the bodies are modelled as rigid and/or deformable bodies, while the joints are modelled through a set of kinematic constraints, that is, the joints are not modelled as contact pairs in the strict sense of the word contact but as algebraic constraints to which implicit forces are associated. The functionality of a joint relies upon the relative motion allowed between the connected components. In most cases, this implies the existence of a clearance between the mating parts, and thus surface contact, shock transmission and the development of different regimes of friction and wear. On the other hand, no

\footnotetext{
* Corresponding author. Tel.: +351 253 510220/238; fax: +351 253516007.

E-mail address: pflores@dem.uminho.pt (P. Flores).
} 
matter how small that clearance is, it can lead to vibration and fatigue phenomena, lack of precision or, even random overall behaviour.

Over the last years, the serious consequences of the joint clearances on the dynamic response of mechanical systems have motivated a number of theoretical investigations on the subject [1-4]. Haines [1] and Ravn [2] presented a literature review concerned with planar motion systems with impacts in revolute joint clearances. These works do not take into account the effects of friction and lubrication. Some others authors, have focused on systems with rigid bodies [4,5]. However, the assumption of rigid bodies can be restrictive for some practical systems [6,7]. Roger and Andrews [8] considered both the dry contact and lubrication effects to perform the dynamic analysis of a slider-crank mechanism with a revolute joint clearance. They treated the bodies as rigid and neglected the hydrodynamic effect due to the wedge-film action. Liu and Lin [9] extended Roger and Andrews' work including both the squeeze-film and wedge-film actions, in which the hydrodynamic forces were evaluated using a numerical iterative procedure. Ravn et al. [10] and Schwab [11] modelled analytically joint clearances in mechanical systems considering both the dry contact and the lubrication effects. More recently, Flores et al. [12] proposed a hybrid model for revolute joint with clearance, in which the dry contact and the squeeze-film effects can be combined in limit situations.

This work focuses upon the dynamics of mechanical systems with revolute joint clearances considering the dry contact, friction and hydrodynamic lubrication effects. Mechanical systems are usually connected by revolute joints, that is, the so-called journal-bearings, in which some clearance is always present. This clearance is necessary to allow relative motion between the connected elements. In the absence of a lubricant, when the journal moves freely within the bearing's boundaries, the forces associated with the journal-bearing are set to be zero. However, when the journal reaches the bearing wall, contact forces are developed in the contact zone, being the dynamics of the journal-bearing controlled by these contact forces. The impact between the two bodies is treated as a continuous event, that is, the local deformations and the contact forces are continuous functions of time. The impact analysis of the system is performed by including the contact forces into the equations of motion during the contact period. The normal contact forces are evaluated as a function of the relative elastic deformation between the colliding bodies coupled with a non-linear viscous-elastic factor representing the energy dissipation during the impact [13]. A modified Coulomb's friction law is used to calculate the tangential friction forces [14].

In order to reduce friction and thus permit easier relative motion between the journal and the bearing, a lubricant is introduced between them for any practical application. Lubricants are widely used in machine elements to reduce friction, avoid any contact between the surfaces, provide load capacity and add stiffness and damping to dissipate undesirable mechanical vibrations. Moreover, the lubricant also prevents corrosion and scavenges heat, dirt and wear debris. The load pressure developed in a hydrodynamic journal-bearing arises from either the flow of a viscous fluid in a converging channel, the wedge-film action, or the resistance of a viscous fluid to being squeezed out from two approaching surfaces, which is known as the squeeze-film action [15]. Many journal-bearings operate under conditions requiring consideration of both wedge and squeeze film effects simultaneously. The hydrodynamic forces that act on the journal-bearing depend on the fluid's properties and on the journal's motion relative to the bearing. Similarly to the models of joint in which only dry contact is considered, the dynamic analysis of the system with lubricated revolute joints is performed by adding the hydrodynamic forces into the system's equations of motion. The hydrodynamic theory for dynamically loaded journal-bearings is used to compute the hydrodynamic forces [15].

The methodologies and procedures adopted throughout this work are presented with the help of a numerical simulation of a slider-crank mechanism with a revolute joint clearance, involving both dry and lubricated models. Based on the results obtained for the different models a discussion of the modelling of clearance revolute joints is presented.

\section{Modelling revolute joints with clearance}

\subsection{Dry contact model}

In the classical analysis of a revolute joint the journal and bearing centres coincide, that is, the revolute joint is considered ideal or perfect. The inclusion of the clearance separates the two centres and, therefore, the joint 
is not ideal anymore. Indeed, from practical point of view, some amount of clearance is always present in revolute joints in order to allow relative motion between the journal and the bearing. Consequently, when compared with an ideal joint modelled by a kinematic constraint two extra degrees of freedom are added to the system for a model with a clearance joint. Fig. 1 depicts a revolute joint with clearance, in which the radial clearance, $c$, is defined as the difference between the bearing and journal radius, $R_{\mathrm{B}}$ and $R_{\mathrm{J}}$, respectively. The nominal journal-bearing length and diameter are represented by $L_{\mathrm{B}}$ and $D_{\mathrm{B}}$, respectively.

It is clear that a revolute joint with clearance does not impose any kinematic constraints on the system, but limits the journal orbit inside the bearing's boundaries. Three different types of motion between the journal and bearing can be observed during the dynamics of the revolute joint clearance, namely, $(i)$ free flight mode, in which the journal moves freely within the bearing's boundaries, that is, the journal and the bearing joint are not in contact; (ii) impact mode, which occurs at the termination of the free flight mode, being impact forces applied to and removed from the system; (iii) permanent contact or following mode, in which contact is always maintained although the relative penetration depth between the bearing and journal varies along the circumference of the journal [12].

If there is no lubricant, the journal can move freely within the bearing until contact between the two bodies takes place. When the journal impacts the bearing wall, normal [13] and tangential [14] contact forces are developed and guide the dynamics of the journal-bearing. These forces are of a complex nature, and their corresponding impulse is transmitted throughout the system. Fig. 2a shows the normal and tangential force components due to an impact between the journal and the bearing. The impact which has both normal
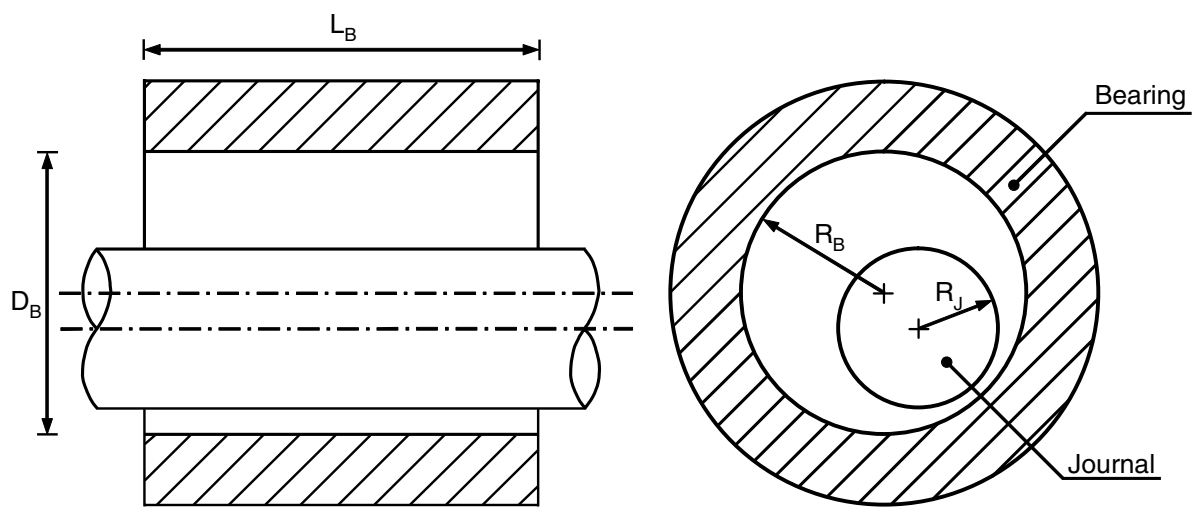

Fig. 1. Revolute joint with clearance. Clearance is exaggerated for illustration.

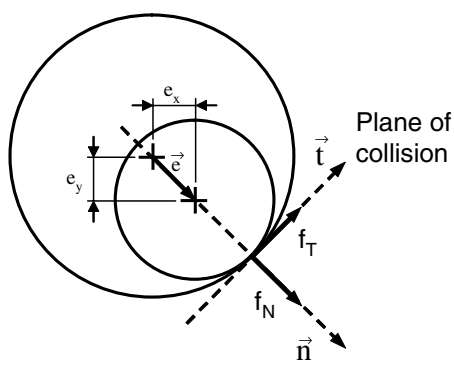

(a)

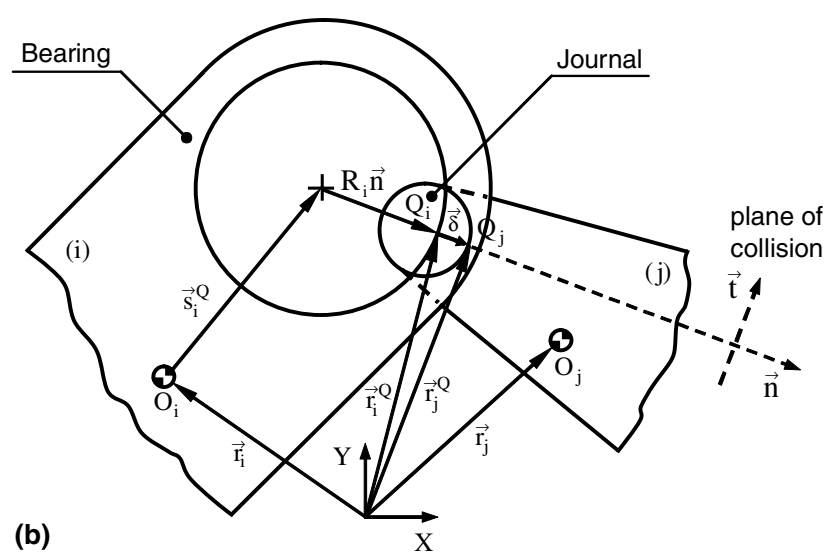

Fig. 2. (a) Normal and tangential forces that act between the journal and bearing in consequence of an impact and (b) representation of a revolute joint with clearance in a mechanical system. 
and tangential relative velocities is treated as an eccentric oblique collision between two bodies. The normal velocity determines whether the contact bodies are approaching or separating. Whereas, the tangential velocity defines whether the bodies are sliding or sticking.

Fig. 2b illustrates a revolute joint with clearance in a mechanical system. The bearing is part of body $i$ and the journal is part of body $j$. For the actual dynamic configuration of the system, the relative penetration depth between the journal and the bearing is expressed as

$$
\delta=e-c>0
$$

where $e$ is the magnitude of the eccentricity vector defined between the bearing and journal centres, and $c$ is the radial clearance, which is a specified parameter. This geometric condition relates the location of the centres of the bearing and journal relative to the radial clearance in the joint. Referring to Fig. 2a, the magnitude of the eccentricity vector is given by,

$$
e=\sqrt{e_{x}^{2}+e_{y}^{2}}
$$

in which $e_{x}$ and $e_{y}$ represent the $X$ and $Y$ components of the displacement of the journal inside the bearing. These relative displacements are obtained from the global position vectors of the bearing and journal centres, respectively.

The journal is considered in free flight motion relative to the bearing until the geometric inequality criterion of Eq. (1) is verified, and, consequently, the contact mode is initialized. Ideally, when $\delta=e-c=0$, the bearing and the journal are in contact to each other. However, due to the computation round-off errors accumulation, a tolerance is introduced in order to accommodate for inaccuracies in the numerical results. Thus, in the present work, the bearing and journal are considered to be in contact when the penetration depth is larger than $1.0 \times 10^{-10}$.

In Fig. 2b points $Q_{i}$ and $Q_{j}$ denote the contact points on the bearing and journal, respectively. The global position of these points is expressed by [4],

$$
\mathbf{r}_{z}^{Q}=\mathbf{r}_{z}+\mathbf{s}_{z}^{Q}+R_{z} \mathbf{n}, \quad(z=i, j)
$$

where $\mathbf{r}_{i}$ and $\mathbf{r}_{j}$ represent the global position of the centres of mass of bodies $i$ and $j, \mathbf{s}_{i}^{Q}$ and $\mathbf{s}_{j}^{Q}$ represent the position of the centres of the journal and bearing relative to the centres of mass of bodies $i$ and $j$, and $R_{i}$ and $R_{j}$ are the bearing and journal radius, respectively. The vector $\mathbf{n}$ is the unit vector normal to the plane of collision, which is defined by

$$
\mathbf{n}=\frac{\mathbf{e}}{\sqrt{e_{x}^{2}+e_{y}^{2}}}
$$

The velocity of the contact points $Q_{i}$ and $Q_{j}$ is found by differentiating Eq. (3) with respect to time, yielding,

$$
\dot{\mathbf{r}}_{z}^{Q}=\dot{\mathbf{r}}_{z}+\dot{\mathbf{s}}_{z}^{Q}+R_{z} \dot{\mathbf{n}}, \quad(z=i, j)
$$

in which the $(\bullet)$ denotes the derivative with respect to time of quantity $(\bullet)$. Thus, the relative contact velocity, necessary to evaluate the contact force, can be defined as

$$
\dot{\boldsymbol{\delta}}=\left(\dot{\mathbf{r}}_{j}-\dot{\mathbf{r}}_{i}\right)+\left(\dot{\mathbf{s}}_{j}^{Q}-\dot{\mathbf{s}}_{i}^{Q}\right)+\left(R_{j}-R_{i}\right) \dot{\mathbf{n}}
$$

The relative contact velocity has to be projected onto the normal and tangential directions in order to determine the components of the normal and tangential velocities, respectively.

In short, the dynamics of a dry journal-bearing is characterized by two different situations. Firstly, when the journal and bearing are not in contact with each other, there are no contact forces associated to the journalbearing. Secondly, when the contact between the two bodies occurs the contact-impact forces are modeled according to a non-linear Hertz's force law (normal force) together with the Coulomb's friction law (tangential force). These two conditions can be expressed as,

$$
\begin{array}{lll}
F=\mathbf{0} & \text { if } & \delta<0 \\
F=F_{\mathrm{N}}+F_{\mathrm{T}} & \text { if } & \delta \geqslant 0
\end{array}
$$


in which, $F_{\mathrm{N}}$ and $F_{\mathrm{T}}$ are normal and tangential force components represented in Fig. 2a, the direction of the forces appearing in Eq. (7) are consistent with their application on the bearing.

Thus, when the journal reaches the bearing wall, that is, the magnitude of the eccentricity vector is greater than radial clearance, an impact takes place and the penetration depth is given by Eq. (1). The impact analysis of the system is performed by including the normal and tangential contact forces into the force vector that appears in the system equations of motion. The suitable numerical models for normal and tangential contact forces are discussed in the following sections.

\subsection{Lubricated model}

When the space between the journal and the bearing is filled with an oil lubricant, a hydrodynamic resistance force develops in opposition to the journal's motion (Fig. 3a). The hydrodynamic force is caused by both squeeze-film and wedge-film actions [15]. In the squeeze-film effect, the pressure generation is due solely to relative radial velocity of the journal-bearing surfaces, that is, the bodies move toward each other. Whereas the wedge-film effect deals with the relative rotational velocity, that is, the fluid is dragged due to the relative angular velocity between the two elements.

When there is an oil lubricant, the forces that act on the journal-bearing depend on the oil's properties, and on the journal motion relative to bearing. These hydrodynamic forces are commonly divided into radial (squeeze-film action) and tangential (wedge-film action) components, as shown in Fig. 3a. Similarly, to the dry contact case, the dynamic analysis of the system with lubricated joint is performed by adding the hydrodynamic forces to the system's equations of motion. The hydrodynamic force model is presented in Section 5 of this work.

In what follows, the fundamental kinematic aspects of a journal-bearing in mechanical systems are presented [16]. With reference to Fig. 3b, the eccentricity vector e, which connects the centres of the bearing and the journal, is calculated as

$$
\mathbf{e}=\mathbf{r}_{j}^{P}-\mathbf{r}_{i}^{P}
$$

where both vectors $\mathbf{r}_{j}^{P}$ and $\mathbf{r}_{i}^{P}$ are expressed in global coordinates with respect to the inertial reference frame $X Y$. The magnitude of the eccentricity vector is evaluated as

$$
e=\sqrt{\mathbf{e}^{\mathrm{T}} \mathbf{e}}
$$

where $\mathbf{e}^{\mathrm{T}}$ denotes the transpose of the vector $\mathbf{e}$.

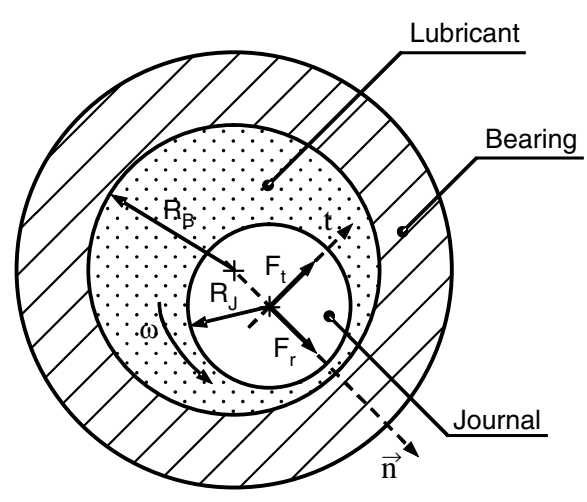

(a)

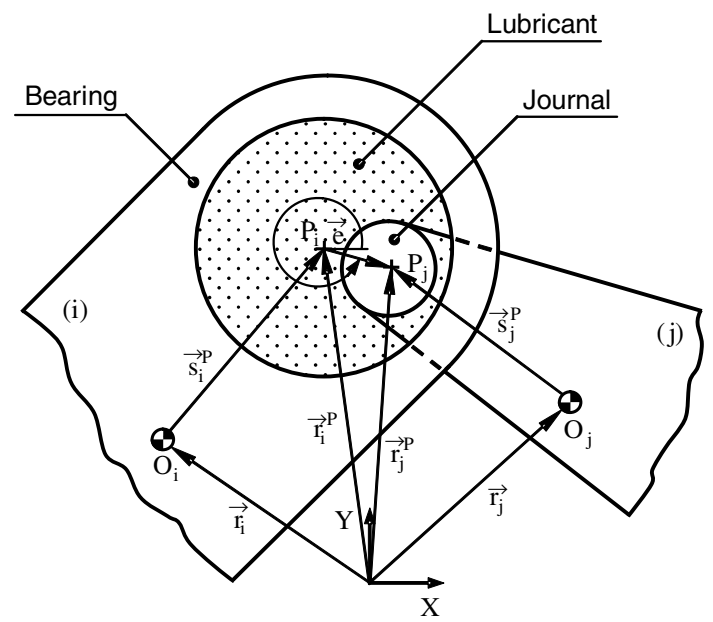

(b)

Fig. 3. (a) Revolute joint clearance with lubricant and representation of radial and tangential hydrodynamic force components and (b) generic configuration of a revolute joint clearance with lubricant in a mechanical system. 
A unit vector, $\mathbf{n}$, along the eccentricity direction is defined as,

$$
\mathbf{n}=\frac{\mathbf{e}}{e}
$$

The direction of the unit vector $\mathbf{n}$ is coincident with the line that connects the centres of the bearing and the journal, denoted here as radial direction. The tangential direction is obtained by rotating vector $\mathbf{n}$ counter clockwise by $90^{\circ}$.

Dividing the eccentricity $e$ by radial clearance $c$ yields the eccentricity ratio $\varepsilon$ written as

$$
\varepsilon=\frac{e}{c}
$$

The time rate of change of eccentricity ratio, denoted by $\dot{\varepsilon}$ is obtained by differentiating Eq. (8) with respect to time, and dividing the result by radial clearance, that is

$$
\dot{\varepsilon}=\frac{\dot{e}}{c}
$$

Due to the direction of the vector $\mathbf{n}$ its components are expressed as function of angle $\gamma$, shown in Fig. $3 b$, as

$$
\left[\begin{array}{c}
\cos \gamma \\
\sin \gamma
\end{array}\right]=\left[\begin{array}{l}
n_{x} \\
n_{y}
\end{array}\right]
$$

where $n_{x}$ and $n_{y}$ are the components of the unit vector $\mathbf{n}$ projected onto the $X$ and $Y$ directions respectively. Thus, Eq. (13) can be re-written as,

$$
\gamma=\operatorname{arctg} \frac{n_{y}}{n_{x}}
$$

Finally, the rate of parameter $\dot{\gamma}$ can be obtained by differentiating Eq. (14) with respect to the time, yielding

$$
\dot{\gamma}=\frac{e_{x} \dot{e}_{y}-\dot{e}_{x} e_{y}}{e^{2}}
$$

The angle $\gamma$ and its rate are used for the hydrodynamic lubrication model discussed in Section 5 of this work.

\section{Elastic-damping contact force model}

Modelling contact forces during an impact plays a crucial role in the dynamic analysis of mechanical systems. The contact force model must be computed by using a suitable constitutive law that takes into account material properties of the colliding bodies, geometric characteristics of the impacting surfaces and the impact velocity. Additionally, the numerical method for the calculation of the contact forces should be stable in order to allow for the integration of the mechanical systems' equations of motion.

Various types of constitutive laws are suggested in the literature, being one of the more prominent proposed by Hertz [17]. However, this law is purely elastic in nature and cannot explain the energy loss during the impact process. Lankarani and Nikravesh [13] overcame this difficulty by separating the normal contact force into elastic and dissipative components as,

$$
F_{\mathrm{N}}=K \delta^{n}+D \dot{\delta}
$$

where the first term represents the elastic forces and the second term accounts for the energy dissipation. In Eq. (16), $K$ is the generalized stiffness parameter, $\delta$ is the relative penetration depth, $D$ is the hysteresis damping coefficient and $\dot{\delta}$ is the relative impact velocity. The exponent $n$ is equal to 1.5 for metallic contacts.

The generalized stiffness parameter $K$ depends on the geometry and physical properties of the contacting surfaces. For two spherical contacting bodies with radii $R_{i}$ and $R_{j}$, the stiffness parameter is expressed by [17],

$$
K=\frac{4}{3\left(\sigma_{i}+\sigma_{j}\right)}\left[\frac{R_{i} R_{j}}{R_{i}+R_{j}}\right]^{\frac{1}{2}}
$$


in which the material parameters $\sigma_{i}$ and $\sigma_{j}$ are given by

$$
\sigma_{z}=\frac{1-v_{z}^{2}}{E_{z}}, \quad(z=i, j)
$$

variables $v_{k}$ and $E_{k}$ are the Poisson's coefficient and the Young's modulus associated with each body, respectively. The radius of curvature is taken as positive for convex surfaces and negative for concave surfaces.

The hysteresis damping coefficient $D$ is expressed by [13],

$$
D=\eta \delta^{n}
$$

where $\eta$ is the hysteretic damping factor. An expression for $\eta$ can be obtained by relating the kinetic energy loss by the impacting bodies to the energy dissipated in the system due to internal damping. Considering these kinetic energies before and after impact, the energy loss $\Delta E$ can be expressed as a function of the restitution coefficient $c_{e}$ and initial impact velocity $\dot{\delta}^{(-)}$as

$$
\Delta E=\frac{1}{2} m \dot{\delta}^{(-)^{2}}\left(1-c_{e}^{2}\right)
$$

The energy loss can also be evaluated by the integration of the contact force around the hysteresis loop. Assuming that the damping force deformation characteristics during the compression and restitution phases are the same, it can be seen that [13]

$$
\Delta E=\oint \mathrm{D} \dot{\delta} \mathrm{d} \delta \approx 2 \int_{0}^{\delta_{\max }} \eta \delta^{n} \dot{\delta} \mathrm{d} a \delta \approx \frac{2}{3} \frac{\eta}{K} m \dot{\delta}^{(-)^{3}}
$$

After substituting Eq. (20) in Eq. (21) an expression for the hysteretic damping factor $\eta$ can be obtained as

$$
\eta=\frac{3 K\left(1-c_{e}^{2}\right)}{4 \dot{\delta}^{(-)}}
$$

which represents the kinetic energy loss of the work done by the contact force.

The hysteretic damping factor expressed by Eq. (22) is now substituted into Eq. (16) resulting in the continuous contact force model, which includes internal damping, written here as,

$$
F_{\mathrm{N}}=K \delta^{n}\left[1+\frac{3\left(1-c_{e}^{2}\right)}{4} \frac{\dot{\delta}}{\dot{\delta}^{(-)}}\right]
$$

This contact force model that accounts for the energy dissipation is found to be satisfactory for general mechanical contacts. Shivaswamy [18] demonstrated experimentally that at low impact velocities, the energy dissipation due to the internal damping is the main contributor to energy loss. The contact force model given by Eq. (23) is only valid for low impact velocities, i.e., speeds that are at the most one order of magnitude lower than the elastic wave travelling velocity.

Other contact models are candidates to be used for the normal contact force and some more insight can be obtained from works that have been developed independently of that described here. In particular the interested reader can find in the works of Kuwabara and Kono [19] and Ramirez et al. [20] good insights on the collision of spheres where the dissipative effects play an important role.

\section{Friction force model}

Friction forces act when contacting bodies tend to slide relative each other. These forces are tangential to the surfaces of contact and are opposite to the sliding velocity. The tangential forces due to the friction phenomenon may be considered when the impact velocity has a relative tangential velocity component, such as in the case of oblique eccentric collisions. Friction is a complex phenomenon which involves interaction between the surfaces of contacting bodies and may lead to different friction regimes such as sliding and stiction [21].

The most fundamental and simplest friction force model is the Coulomb's friction law [22]. This model assumes that the friction force between sliding bodies with respect to each other is proportional to the normal 


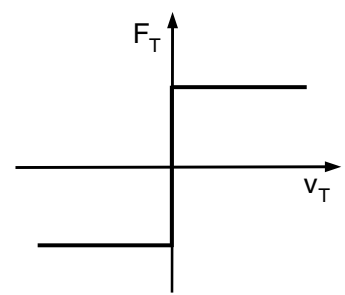

(a)

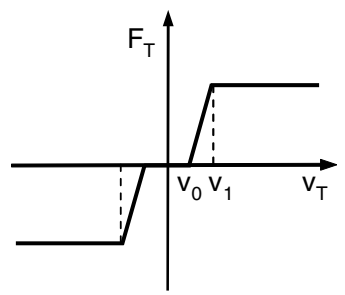

(b)

Fig. 4. (a) Standard Coulomb's friction law and (b) modified Coulomb's friction law.

contact force. The constant of proportionality is called the friction coefficient. The definition of the Coulomb's friction law poses numerical difficulties where the relative tangential velocity is in the vicinity of zero. The problems arise during the integration process because the friction force changes instantaneously from $-F_{\mathrm{T}}$ to $+F_{\mathrm{T}}$, as pictured in Fig. $4 \mathrm{a}$, for small positive and negative numbers of the relative velocity, which is perceived by the integration algorithm as a high frequency content in the dynamic response that leads to successive decreases in the integration time step. Therefore, a continuous friction force-velocity relationship compatible with the requirement for a stable integration of the system dynamic equations and with acceptable precision is required.

A modified friction force model that fulfils the needs identified, presented by Ambrósio [14], is expressed as

$$
F_{\mathrm{T}}=-c_{\mathrm{f}} c_{\mathrm{d}} F_{\mathrm{N}} \frac{\vec{v}_{\mathrm{T}}}{\left|\vec{v}_{\mathrm{T}}\right|}
$$

where $c_{\mathrm{f}}$ is the friction coefficient, $F_{\mathrm{N}}$ is the normal contact force and $\vec{v}_{\mathrm{T}}$ is the relative tangential velocity. The dynamic correction coefficient $c_{\mathrm{d}}$ is given by

$$
c_{\mathrm{d}}=\left\{\begin{array}{lll}
0 & \text { if } & \left|\vec{v}_{\mathrm{T}}\right| \leqslant v_{0} \\
\frac{\left|\vec{v}_{\mathrm{T}}\right|-v_{0}}{v_{1}-v_{0}} & \text { if } & v_{0} \leqslant\left|\vec{v}_{\mathrm{T}}\right| \leqslant v_{1} \\
1 & \text { if } & \left|\vec{v}_{\mathrm{T}}\right| \geqslant v_{1}
\end{array}\right.
$$

in which, $v_{0}$ and $v_{1}$ are given tolerances for the tangential velocity. This dynamic correction factor effectively prevents that the friction force changes direction for almost null values of the tangential velocity. Therefore, the great merit of the modified Coulomb's law expressed by Eq. (24) is that it allows the numerical stabilization of the integration algorithm. This friction force model, which is schematically illustrated in Fig. 4b, does not account for other tribological phenomena like the adherence between the sliding contact surfaces, i.e., stiction.

\section{Hydrodynamic force model}

The Reynolds' equation is used to evaluate the hydrodynamic forces developed by the fluid pressure field in a journal-bearing. The isothermal Reynolds' equation for a dynamically loaded journal-bearing for which the fluid is incompressible and the journal and bearing do not experience any elastic deformation, can be written as [15],

$$
\frac{\partial}{\partial X}\left(\frac{h^{3}}{\mu} \frac{\partial p}{\partial X}\right)+\frac{\partial}{\partial Z}\left(\frac{h^{3}}{\mu} \frac{\partial p}{\partial Z}\right)=6 U \frac{\partial h}{\partial X}+12 \frac{\mathrm{d} h}{\mathrm{~d} t}
$$

where $h$ is the fluid film thickness, $p$ is the fluid pressure, $\mu$ is the absolute fluid viscosity, $U$ is the relative tangential velocity, and $X$ and $Z$ are the radial and axial directions, respectively. The two terms on the right-hand side of Eq. (26) represent the two different effects of pressure generation on the lubricant film, that is, wedge and squeeze film actions, respectively.

It is known that the Reynolds' equation (26) is a non-homogeneous partial differential of elliptical type being the exact solution difficult to obtain and, in general, requiring considerable numerical effort. However, 
it is possible to solve the Reynolds' equation analytically when either the first or the second term on the lefthand side is null. These particular solutions correspond to models of infinitely-short and infinitely-long journal-bearing, respectively. For an infinitely-long journal-bearing it is assumed a constant fluid pressure and a negligible leakage in the $Z$-direction. In many cases it is possible to treat a journal-bearing as infinitely-long and consider only its middle point. This solution, firstly presented by Sommerfeld [15], is valid for length-todiameter ratios $L_{\mathrm{B}} / D_{\mathrm{B}}$ greater than 2 . Considering the case of an infinitely long journal-bearing the Reynolds' equation is simplified as [15]

$$
\frac{\partial}{\partial X}\left(\frac{h^{3}}{\mu} \frac{\partial p}{\partial X}\right)=6 U \frac{\partial h}{\partial X}+12 \frac{\mathrm{d} h}{\mathrm{~d} t}
$$

By integrating Eq. (27), the pressure filed in the journal-bearing is given by

$$
p=6 \mu\left(\frac{R_{\mathrm{J}}}{c}\right)^{2}\left\{\frac{(\omega-2 \dot{\gamma})(2+\varepsilon \cos \theta) \varepsilon \sin \theta}{\left(2+\varepsilon^{2}\right)(1+\varepsilon \cos \theta)^{2}}+\frac{\dot{\varepsilon}}{\varepsilon}\left[\frac{1}{(1+\varepsilon \cos \theta)^{2}}-\frac{1}{(1+\varepsilon)^{2}}\right]\right\}
$$

where $c$ is the radial clearance, $\theta$ is the angular coordinate, $\varepsilon$ is the eccentricity ratio, $\dot{\varepsilon}$ is the time rate of change of eccentricity ratio, $\mu$ is the dynamic lubricant viscosity and $R_{\mathrm{J}}$ is the journal radius.

Eq. (28) enables the calculation of the pressure distribution in a hydrodynamic infinitely-long loaded journal-bearing as function of the dynamic journal-bearing parameters and geometry. However, it is convenient to determine the force components of the resultant pressure field in the directions aligned and perpendicular to the line of centres of the journal and bearing. These force components can be obtained by integration of the pressure field around half domain $\pi$, that is, the pressure distribution is integrated only over the positive region by setting the pressure in the remaining portion equal to zero. These are known as the Gümbel's boundary conditions. This analysis involves a great deal of mathematical manipulation for which the interested reader is referred to Ref. [15]. Thus, the component forces along eccentricity direction and its perpendicular dircection are, for $\dot{\varepsilon}>0$, given by [15],

$$
\begin{aligned}
& F_{\mathrm{r}}=-\frac{\mu L_{\mathrm{B}} R_{\mathrm{J}}^{3}}{c^{2}} \frac{6 \dot{\varepsilon}}{\left(2+\varepsilon^{2}\right)\left(1-\varepsilon^{2}\right)^{3 / 2}}\left[4 k \varepsilon^{2}+\left(2+\varepsilon^{2}\right) \pi \frac{k+3}{k+3 / 2}\right] \\
& F_{\mathrm{t}}=\frac{\mu L_{\mathrm{B}} R_{\mathrm{J}}^{3}}{c^{2}} \frac{6 \pi \varepsilon(\omega-2 \dot{\gamma})}{\left(2+\varepsilon^{2}\right)\left(1-\varepsilon^{2}\right)^{1 / 2}} \frac{k+3}{k+3 / 2}
\end{aligned}
$$

and for $\dot{\varepsilon}<0$

$$
\begin{aligned}
& F_{\mathrm{r}}=-\frac{\mu L_{\mathrm{B}} R_{\mathrm{J}}^{3}}{c^{2}} \frac{6 \dot{\varepsilon}}{\left(2+\varepsilon^{2}\right)\left(1-\varepsilon^{2}\right)^{3 / 2}}\left[4 k \varepsilon^{2}-\left(2+\varepsilon^{2}\right) \pi \frac{k}{k+3 / 2}\right] \\
& F_{\mathrm{t}}=\frac{\mu L_{\mathrm{B}} R_{\mathrm{J}}^{3}}{c^{2}} \frac{6 \pi \varepsilon(\omega-2 \dot{\gamma})}{\left(2+\varepsilon^{2}\right)\left(1-\varepsilon^{2}\right)^{1 / 2}} \frac{k}{k+3 / 2}
\end{aligned}
$$

where the parameter $k$ is defined as

$$
k^{2}=\left(1-\varepsilon^{2}\right)\left[\left(\frac{\omega-2 \dot{\gamma}}{2 \dot{\varepsilon}}\right)^{2}+\frac{1}{\varepsilon^{2}}\right]
$$

in which $L_{\mathrm{B}}$ is the journal-bearing length, $c$ is the radial clearance, $\omega$ is the relative angular velocity between the journal and the bearing and $\gamma$ is the angle between the eccentricity direction and the $X$-axis, as depicted by Fig. 3b. The notation $(\bullet)$ denotes the time derivative of the quantity $(\bullet)$.

The hydrodynamic force components given by Eqs. (29)-(33) are non-linear functions of the time parameters, $\omega, \varepsilon, \dot{\varepsilon}, \gamma$, and $\dot{\gamma}$, which can be evaluated at any instant of time from the dynamic analysis of the mechanical system. The solution of these equations presents no problem as the motion of the journal's centre is obtained from the dynamic analysis of the overall mechanical system.

In traditional tribology analysis of journal-bearings the applied forces are known and the motion of the journal centre inside the bearing's boundaries is evaluated by solving the differential equations for the time 
dependent variables. However, in the present work instead of knowing the applied load, the relative journalbearing motion characteristics are known. The fluid force resulting from the pressure distribution in the lubricant is then calculated. Thus, since all the state of variables are known from dynamic analysis of the mechanical system, the hydrodynamic forces given by Eqs. (29)-(33) are evaluated and introduced as external generalized forces into the equations of motion of the mechanical system.

\section{Application case: slider-crank mechanism}

The slider-crank mechanism is chosen to demonstrate the methodologies presented in this work. The mechanism under consideration is made of four rigid bodies, two ideal revolute joints and one perfect translational joint. A revolute joint with clearance exists between the connecting rod and slider, as shown in Fig. 5. Due to the presence of the non-ideal joint this mechanism has three degrees of freedom. The acceleration due to gravity is taken as acting in the negative $Y$-direction and the mechanism is defined as moving in a vertical plane.

The set of data adopted to build the model used in the dynamic simulations is listed in Table 1 .

At the start of the dynamic analysis, the crank and connecting rod are aligned in the $X$-direction, corresponding to the top dead point. The initial conditions for the simulation include the crank speed of $5000 \mathrm{rpm}$, which is maintained constant during the simulations. Furthermore, the remaining initial conditions, necessary to start the dynamic analysis, are obtained from kinematic simulation of the slider-crank mechanism in which all the joints are considered to be ideal. The remaining parameters used for the simulation are listed in Table 2. The radial clearance is equal to $0.05 \mathrm{~mm}$, which corresponds to the actual clearance size in a typical journal-bearing with dimensions used the present work. For example, for a journal-bearing in which the nominal diameter is between 18 and $30 \mathrm{~mm}$ the minimum and maximum recommended clearance sizes are 0.02 and 0.06 , respectively [23].

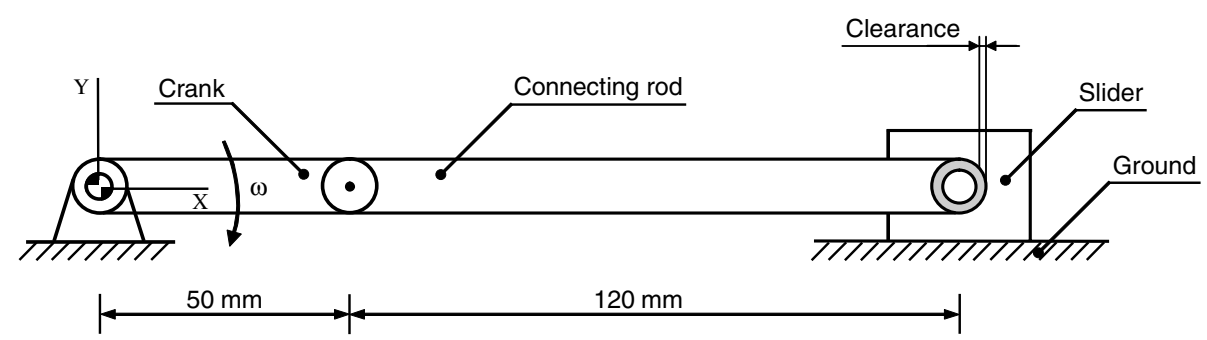

Fig. 5. Slider-crank mechanism with a revolute joint clearance between the connecting rod and slider. This picture corresponds to the initial simulation configuration.

Table 1

Geometric and inertia data of the slider-crank mechanism

\begin{tabular}{llll}
\hline Body & Length $(\mathrm{m})$ & Mass $(\mathrm{kg})$ & Moment of inertia $\left.(\mathrm{kg} \mathrm{m})^{2}\right)$ \\
\hline Crank & 0.05 & 0.30 & 0.00001 \\
Connecting rod & 0.12 & 0.21 & 0.00025 \\
Slider & - & 0.14 & - \\
\hline
\end{tabular}

Table 2

Parameters used in the dynamic simulation for the slider-crank mechanism

\begin{tabular}{llll}
\hline Bearing radius & $10.0 \mathrm{~mm}$ & Poisson's ratio & 0.3 \\
Restitution coefficient & 0.9 & Dynamic fluid viscosity & $400 \mathrm{cP}$ \\
Friction coefficient & 0.1 & Baumgarte coefficient- $\alpha, \beta$ & 5 \\
Material coefficient & 1.5 & Integration step size & $0.00001 \mathrm{~s}$ \\
Young's modulus & $207 \mathrm{GPa}$ & Integration tolerance & 0.000001 \\
\hline
\end{tabular}


The performance of the slider-crank mechanism is quantified by plotting the reaction forces in the joint with clearance and the reaction moment that acts on the crank. Additionally, the trajectories of the journal inside the boundaries of the bearing and the maps that relates the velocities with accelerations are plotted. These plots help to appraise the global dynamic response of the mechanism. The global results reported correspond to two full crank cycles obtained after the system reaches the steady-state condition, in order to ensure the inexistence of other transient effects that are not due to the joint clearance. The results for this mechanism are compared with the dynamic behaviour of the slider-crank mechanism with ideal joints only.

Three different situations are considered in the analysis carried hereafter. Firstly, the revolute joint clearance is modelled as a dry contact joint without friction. Secondly, the friction forces developed at the dry joint clearance are also included in the first model. Finally, the joint clearance is modelled as a lubricated joint, being the oil fluid a SAE 40 multigrade that is used in small combustion engines, and for which the dynamic viscosity is approximately equal to $400 \mathrm{cP}$ for an operating temperature of $40^{\circ}$.

For the simulation of the dry contact the continuous approach for the evaluation the contact force is applied and the energy dissipation in form of hysteresis damping is given by Eq. (23). The friction forces are calculated using a modified Coulomb's friction law depicted by Eq. (24). For the lubricated simulations, the hydrodynamic theory for dynamically loaded journal-bearings, described by Eqs. (29)-(33) is used to compute the hydrodynamic forces (Eqs. (29)-(33)).

The equations of motion for a dynamic system subjected to holonomic constraints can be stated in the form [24],

$$
\left[\begin{array}{ll}
\mathbf{M} & \boldsymbol{\Phi}_{q}^{\mathrm{T}} \\
\boldsymbol{\Phi}_{q} & \mathbf{0}
\end{array}\right]\left\{\begin{array}{l}
\ddot{\mathbf{q}} \\
\boldsymbol{\lambda}
\end{array}\right\}=\left\{\begin{array}{c}
\mathbf{g} \\
\boldsymbol{\gamma}^{\#}-2 \alpha \dot{\boldsymbol{\Phi}}-\beta^{2} \mathbf{\Phi}
\end{array}\right\}
$$

where $\mathbf{M}$ is the global system mass matrix, containing the mass and moments of inertia of all bodies, $\boldsymbol{\Phi}_{q}$ is the Jacobian of constraint equations, $\boldsymbol{\Phi}_{q}^{\mathrm{T}}$ is the transpose of matrix $\boldsymbol{\Phi}_{q}$, the vector $\ddot{\mathbf{q}}$ contains the generalized state accelerations, the vector $\lambda$ holds the Lagrange multipliers, $\mathbf{g}$ is the vector of generalized forces which contains the external applied forces as well as the forces developed at the joint clearances, $\gamma^{\#}$ is the vector of quadratic velocity terms, $\boldsymbol{\Phi}$ is the vector of kinematic constraints, $\dot{\boldsymbol{\Phi}}$ is the constraint velocity equation. The Baumgarte stabilization method [25], with the coefficients $\alpha$ and $\beta$ is used to ensure the stabilization of the constraint violations associated with the kinematic constraints of the ideal joints. Given the initial conditions for position and velocity, the numeric equations of motion (34) are solved for acceleration vector, $\ddot{\mathbf{q}}$, and Lagrange multipliers, $\lambda$. The resulting accelerations together with the velocities are then integrated in time to obtain the velocities and positions at the next time step. This basic procedure is repeated until the final simulation time interval is reached. Note that the equations may exhibit a stiff behaviour, and therefore, integration algorithms such as the one proposed by Gear [26] are preferred for the numerical solution of the problem.

Fig. 6 shows the reaction force for the joint with the clearance in the three different modes. When the force in the joint with clearance is represented by the dry contact model, with or without friction, the reaction force presents peaks that have a magnitude comparable to what is observed in an ideal joint, as shown in Fig. 6a and b. These peaks are originated by the contact-impact forces developed during the contact between the journal and the bearing. Furthermore, the non-smooth evolution in the reaction force curves suggests that the system response is non-periodic and close to have a chaotic nature. In sharp contrast with to the dry contact simulations, when the force in the joint with clearance is modelled with the lubrication force model, the reaction force curve, plotted in Fig. 6c, is much smoother and closer to the behaviour observed for an ideal joint, being the system response periodic. This means that the fluid between the journal and bearing surfaces acts in a same way of a non-linear spring-damper element in the system that avoids the metal-to-metal contact, and forces the journal to move away from the bearing wall.

Fig. 7 illustrates how the joint clearance model influences the crank driving moment, that is, the moment necessary to maintain constant the crank angular velocity during the simulation. Since in the present work all the links of the slider-crank mechanism are considered as rigid bodies, the reaction forces developed at the joint with clearance are transmitted throughout the system causing the crank moment to fluctuate. This effect is visible by the peaks on the force curves obtained in the numerical simulations that use the dry contact model, with and without friction. For the case simulated with lubricated model, the crank moment is of 

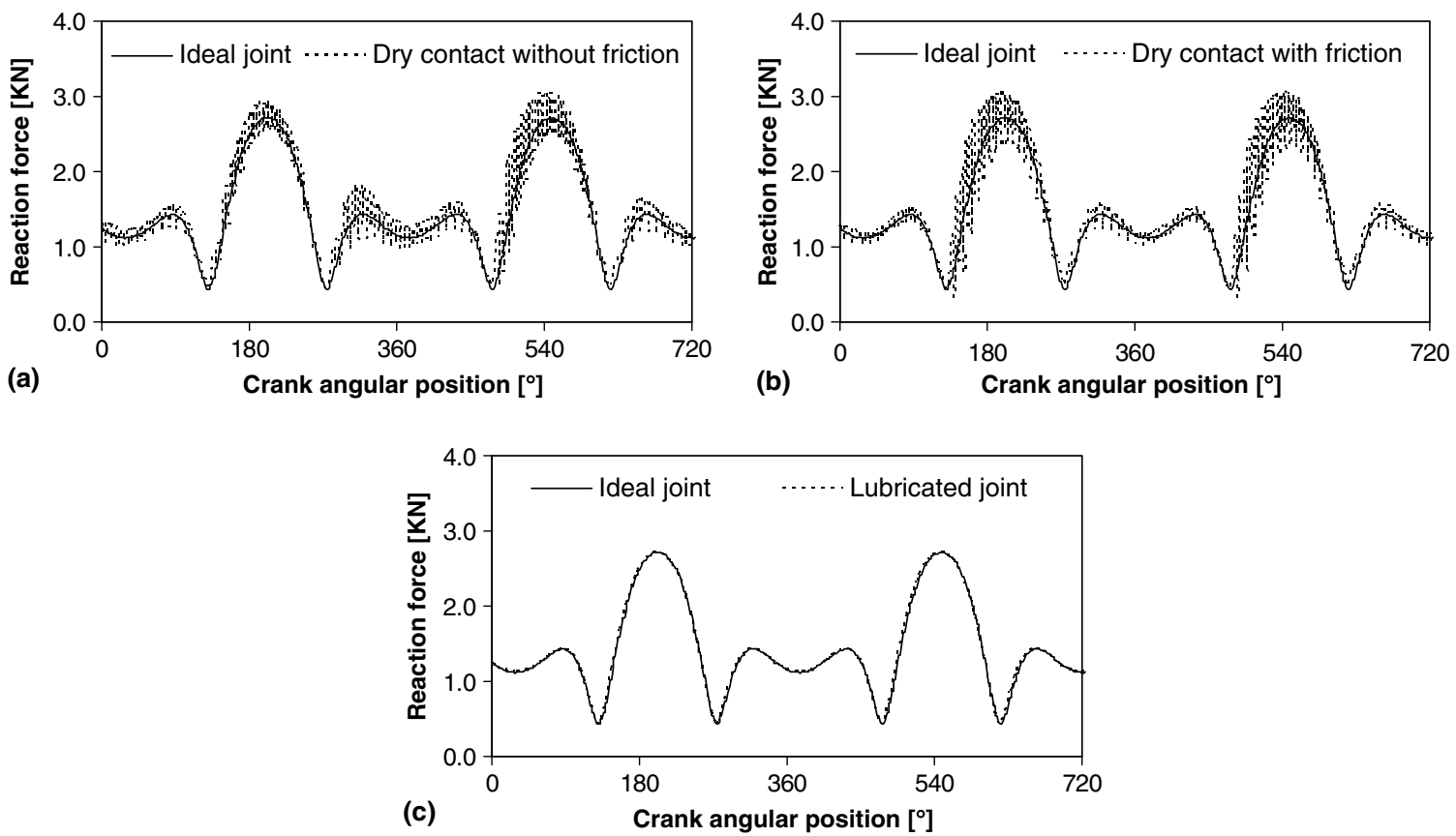

Fig. 6. Reaction force at the joint clearance for the different simulations: (a) dry contact model without friction, (b) dry contact model with friction and (c) lubricated joint model.
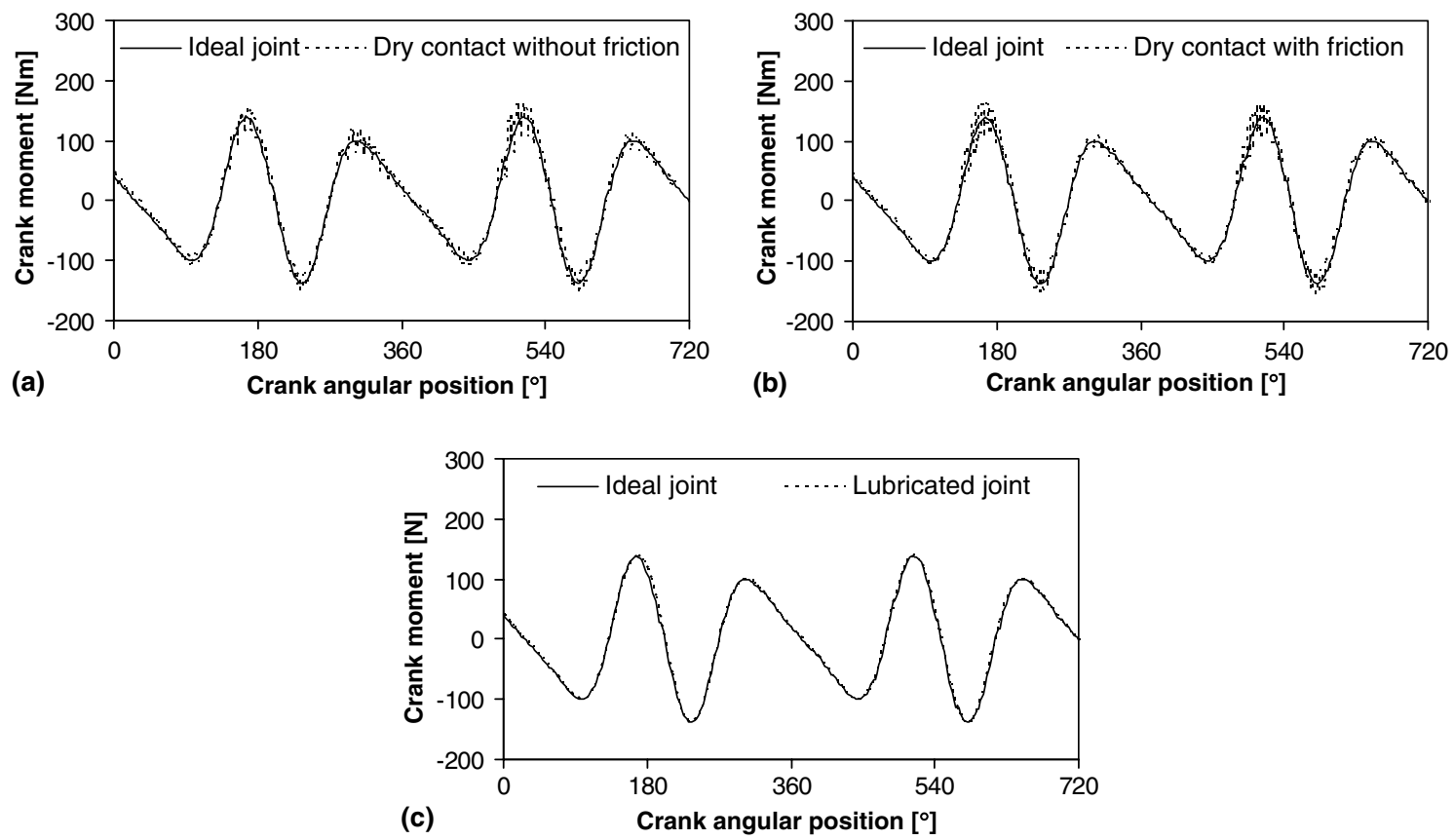

Fig. 7. Driving moment on the crank for the different simulations: (a) dry contact model without friction, (b) dry contact model with friction and (c) lubricated joint model.

the same order of magnitude of the moment obtained with the ideal joint model shown in Fig. 7c. The fluid absorbs some of the energy produced by the slider when it accelerates or decelerates. Therefore, part of this 


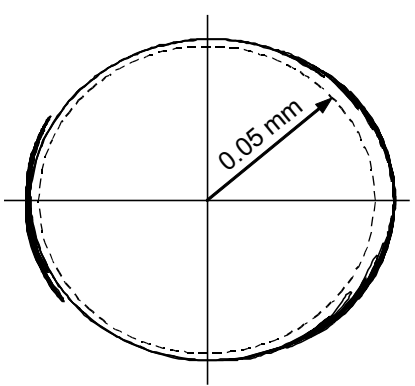

(a)

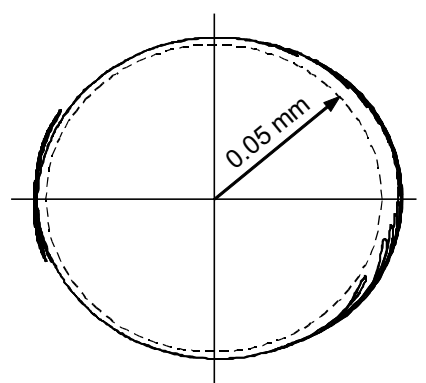

(b)

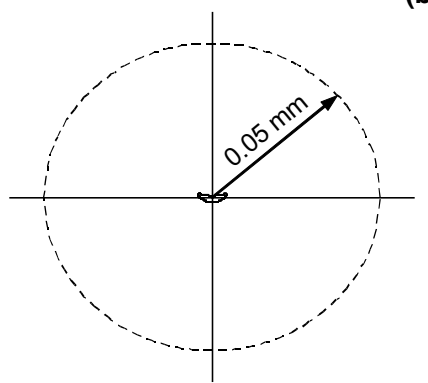

(c)

Fig. 8. Journal centre trajectory for the different simulations: (a) dry contact model without friction, (b) dry contact model with friction and (c) lubricated joint model.
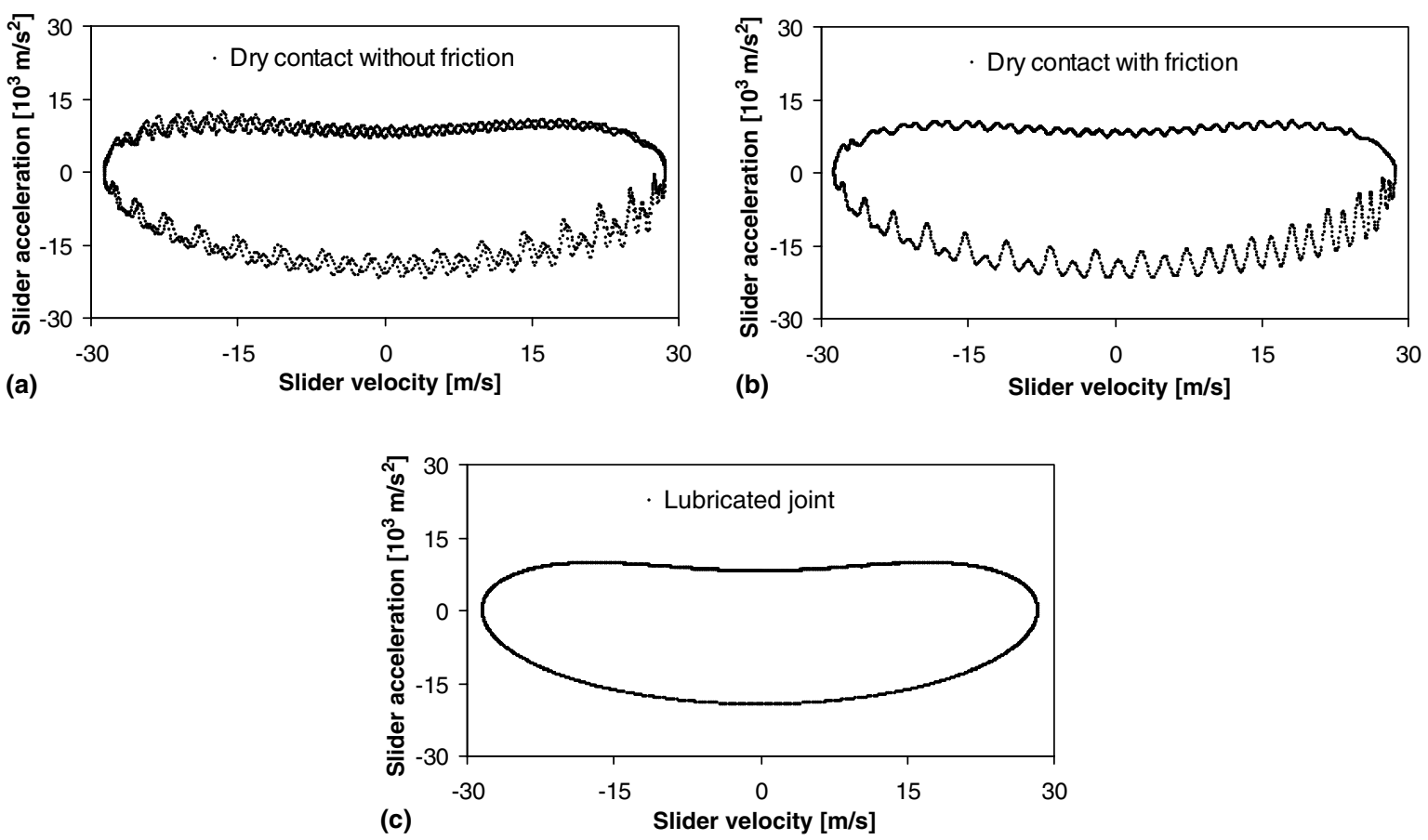

Fig. 9. Maps relating velocities with accelerations for the different simulations: (a) dry contact model without friction, (b) dry contact model with friction and (c) lubricated joint model.

energy is not transmitted to the crank, which results in lower reaction moments in the lubricated model of the joint with clearance when compared to the outcome of the model with ideal joints only. 
As far as the journal centre trajectory relative to the bearing centre is concerned, two different situations can be observed. First, for both dry contact models with and without friction, the journal is always in contact with the bearing wall, in which the penetration depth, and, consequently, the contact force, varies along the radial direction, as shown in Fig. 8a and b. However, for the simulation with lubricated model, the journal centre orbit is very close to the bearing centre, which means that the journal and bearing are always apart from each other never breaking the fluid film. This observation supports the characterization of the global system response as periodic and the fact that it is quite similar to the response for the model with ideal joints only.

The maps described in Fig. 9 are employed to qualitatively study the global dynamic response of the slidercrank mechanism when the three different models for the joint clearance are used. These maps are used here to describe the relation between the velocity and acceleration of the slider. By observing Fig. 9a, for the system model without friction, it is clear that the system response is not periodic as the transformation represented in the map shows that different cycles of the mechanism lead to different curves in the map. The inclusion of friction leads to better response of the system, but without ensuring its periodicity, because the motion of the system does not repeat from cycle to cycle (Fig. 9b). Fig. 9c shows the map for the simulation with a lubricated joint model. The smooth curve shape indicates that the system response is regular and periodic. It should be highlighted that a mechanism with ideal joints has a map very similar to that of the lubricated joint.

The overall results presented with the application are in line with those of published works on this field for the case that includes the dry contact models [2,7,27]. As far as the lubricated joint results are concerned, the global results are also in line with those offered in the literature [10].

\section{Conclusions and future developments}

The influence of the joint clearances in the response of models of mechanical systems was investigated. The joints were modelled considering their actual characteristics that include clearance, friction and lubrication. The methodologies and procedures adopted in this work were applied to a slider-crank mechanism in which a revolute joint with clearance is used between the slider and connecting rod. This application case makes an extensive use of a multibody system formulation with rigid bodies connected by kinematic joints with contactimpact and hydrodynamic force models.

Three different models for the joint clearance have been analyzed, namely, dry contact without friction, dry contact with friction effect and lubricated model.

The numerical results presented in this work, emphasize the fact that in the dry contact models, there are high peaks in the magnitude reaction force and in the crank reaction moment that lead to vibrations on the mechanism, joint wear and, eventually, the fatigue problems. The dynamic system response is basically characterized as non-periodic. But, when the joint clearance is modelled as a lubricated journal-bearing, the reaction force and the crank moment variations are very smooth, resembling those obtained for models with ideal joints only. The fluid lubricant acts like a non-linear spring-damper element that introduces damping and stiffness to the system and avoids the metal-to-metal contact. The lubricant damping effect absorbs part of the kinetic energy and produces smoother and periodic system responses. Indeed, lubricants are introduced in between the journal and bearing in order to separate the surfaces and reduce friction, which would otherwise cause large amounts of wear. In short, for actual joints, the clearance, friction and lubrication phenomena are always present and can significantly alter the dynamic responses of the system, consequently, the ability to model these phenomena plays an important role in predicting accurately the dynamic responses of the mechanical systems. From this point of view all the models proposed are able to capture the different phenomena involved in the dynamics of multibody systems with realistic revolute joints.

\section{Acknowledgements}

The research work presented in this paper was supported by Fundação para a Ciência e a Tecnologia, partially financed by Fundo Comunitário Europeu FEDER, under project POCTI/2001/EME/38281 entitled 'Dynamic of Mechanical Systems with Joint Clearances and Imperfections'. 


\section{References}

[1] R.S. Haines, Survey: 2-dimensional motion and impact at revolute joints, Mechanism and Machine Theory 15 (1980) $361-370$.

[2] P. Ravn, A continuous analysis method for planar multibody systems with joint clearance, Multibody System Dynamics 2 (1998) 124.

[3] K. Soong, B.S. Thompson, A theoretical and experimental investigation of the dynamic response of a slider-crank mechanism with radial clearance in the gudgeon-pin joint, Journal of Mechanical Design 112 (1990) 183-189.

[4] P. Flores, J. Ambrósio, Revolute joints with clearance in multibody systems, Computers and Structures 82 (17-19) (2004) 1359-1369.

[5] S. Dubowsky, F. Freudenstein, Dynamic analysis of mechanical systems with clearances Part 1: formulation of dynamic model, Journal of Engineering for Industry Series B 93 (1) (1971) 305-309.

[6] K. Soong, An analytical and experimental study of the elastodynamic response characteristics of planar linkage mechanism with bearing clearances, Ph.D. Dissertation, Michigan State University, Michigan, USA, 1988.

[7] A.L. Schwab, J.P. Meijaard, P. Meijers, A comparison of revolute joint clearance model in the dynamic analysis of rigid and elastic mechanical systems, Mechanism and Machine Theory 37 (9) (2002) 895-913.

[8] R.J. Roger, G.C. Andrews, Dynamic simulation of planar mechanical systems with lubricated bearing clearances using vectornetwork methods, Journal of Engineering for Industry 99 (1) (1977) 131-137.

[9] T.S. Liu, Y.S. Lin, Dynamic analysis of flexible linkages with lubricated joints, Journal of Sound and Vibration 141 (2) (1990) 193205.

[10] P. Ravn, S. Shivaswamy, B.J. Alshaer, H.M. Lankarani, Joint clearances with lubricated long bearings in multibody mechanical systems, Journal of Mechanical Design 122 (2000) 484-488.

[11] A.L. Schwab, Dynamics of flexible multibody systems, small vibrations superimposed on a general rigid body motion, Ph.D. Dissertation, Delft University of Technology, Netherlands, 2002.

[12] P. Flores, J. Ambrósio, J.C. Claro, Dynamic analysis for planar multibody mechanical systems with real joints, in: J. Ambrósio (Ed.), Proceedings of ECCOMAS Thematic Conference on Advances in Computational Multibody Dynamics 2003, Lisbon, Portugal, July $1-4,2003,26 \mathrm{p}$.

[13] H.M. Lankarani, P.E. Nikravesh, A contact force model with hysteresis damping for impact analysis of multibody systems, Journal of Mechanical Design 112 (1990) 369-376.

[14] J. Ambrósio, Impact of rigid and flexible multibody systems: deformation description and contact models, in: W. Schiehlen, M. Valásek (Eds.), Proceedings of the NATO-ASI on Virtual Non-linear Multibody Systems, vol. II, Prague, Czech Republic, June 23, July 3, 2002, pp. 15-33.

[15] O. Pinkus, S.A. Sternlicht, Theory of Hydrodynamic Lubrication, McGraw-Hill, New York, 1961.

[16] P. Flores, H.M. Lankarani, J. Ambrósio, J.C.P. Claro, Modeling lubricated revolute joints in multibody mechanical systems, Proceedings of the Institution of Mechanical Engineers, Part-K, Journal of Multi-body Dynamics 218 (2004) $183-190$.

[17] H. Hertz, On the contact of solids, 1881, On the contact of rigid elastic solids and on hardness, 1882, Translated by D.E. Jones, G.A. Schott, Miscellaneous Papers, MacMillan and Co. Ltd., London, 1896, pp. 146-183.

[18] S. Shivaswamy, Modeling contact forces and energy dissipation during impact in multibody mechanical systems, Ph.D. Dissertation, Wichita State University, Wichita, USA, 1997.

[19] G. Kuwabara, K. Kono, Restitution coefficient in a collision between two spheres, Japanese Journal of Applied Physics 26 (8) (1987) $1230-1233$.

[20] R. Ramirez, T. Poschel, N. Brilliantov, T. Schwager, Coefficient of restitution of colliding viscolelastic spheres, Physical Review E 60 (4) (1999) 4465-4472.

[21] S. Ahmed, H.M. Lankarani, M.F.O.S. Pereira, Frictional impact analysis in open-loop multibody mechanical system, Journal of Mechanical Design 121 (1999) 119-127.

[22] D.T. Greenwood, Principles of Dynamics, Prentice-Hall, Englewood Cliffs, New Jersey, 1965.

[23] L.V. Cunha, Desenho Técnico, 8a edição, Fundação Calouste Gulbenkian, Lisboa, 1990.

[24] P.E. Nikravesh, Computer-Aided Analysis of Mechanical Systems, Prentice-Hall, Englewood Cliffs, New Jersey, 1988.

[25] J. Baumgarte, Stabilization of constraints and integrals of motion in dynamical systems, Computer Methods in Applied Mechanics and Engineering 1 (1972) 1-16.

[26] C.W. Gear, Numerical Initial Value Problems in Ordinary Differential Equations, Prentice-Hall, Englewood Cliffs, New Jersey, 1971.

[27] J. Rhee, A. Akay, Dynamic response of a revolute joint with clearance, Mechanism and Machine Theory 31 (1) (1996) 121-134. 